\title{
Statistical Analysis of College students' Common Diseases in Tianjin Agricultural University from Different Seasons
}

\author{
Hongjun Teng ${ }^{1, a^{*}}$, Zhenbo Bao ${ }^{2, b}$ and Fan Yang ${ }^{2, c}$ \\ ${ }^{1}$ Clinic, Tianjin Agricultural University, Tianjin, China, 300384 \\ ${ }^{2}$ Engineering and Technology College, Tianjin Agricultural University, Tianjin, China, 300384 \\ a*hongjun-teng@163.com, bbaozhenbo@sohu.com, 'cfanyang20062800@126.com
}

Keywords: College students; Common diseases; Statistical Analysis; Different Seasons

\begin{abstract}
College students shoulder the heavy responsibility of building the country. College students' physical health has a profound influence on the development of the country. It is an important task for higher education to continuously improve college students' physical health. From September 16th, 2016 to September 15th, 2017, statistics were made on the types, numbers, and proportions of college students' common diseases in Tianjin Agricultural University from different seasons, and the common disease patterns of college students in different seasons were summarized. The number of common diseases in the spring (364 cases) is the highest and the incidence rate is the highest. Considering the time of winter and summer vacations, the incidence rate was higher in summer and winter, and the incidence was lowest in autumn ( 233 cases). The four most common diseases that have the highest incidence rate throughout the year are cold, upper respiratory tract infections (pharyngitis, tonsillitis, etc.), sprains and fire. The incidence of common diseases such as skin allergies, gastroenteritis, bronchitis, toothache, conjunctivitis, stomachache and eye fatigue is summarized. The statistical results provide some references for school health educators to better carry out student health education and reduce the incidence of college students' common diseases.
\end{abstract}

\section{Introduction}

College students are an important resource for scientific and technological progress, college students shoulder the heavy responsibility of building the country, and their physical health has profound influence on society $[1,2]$. I have been engaged in the diagnosis and treatment of comprehensive out-patient clinics, health care, and health education for more than 10 years. I am deeply aware of the laws governing common diseases among college students, have targeted health education, and promote college students' knowledge of disease prevention and health care, which can effectively reduce the occurrence of college students' common diseases [3, 4]. From September 16, 2016 to September 15, 2017, a statistical analysis of college students' common diseases is conducted $[5,6]$. This provides a reference for better work on preventive health care and health education [7, 8].

\section{Statistical Analysis of College students' Common Diseases in Autumn in Tianjin Agricultural University}

From September 16, 2016 to November 15, 2016(Autumn), statistics were conducted on the college students' common diseases treated by out-patients at Tianjin Agricultural University Hospital. The statistical results are shown in Table 1. In the autumn, there are 233 common disease patients, accounting for more than $5 \%$ of the common diseases are cold, upper respiratory infections (pharyngitis, tonsils, etc.), fire, sprains, skin allergies and toothache. 
Table 1 The distribution and proportion of college students' common diseases in autumn in Tianjin Agricultural University

\begin{tabular}{|c|c|c|c|}
\hline No. & Common diseases & Cases & Proportion[\%] \\
\hline 1 & Cold & 74 & 31.76 \\
\hline 2 & Upper respiratory tract infections (pharyngitis, tonsils, etc.) & 37 & 15.88 \\
\hline 3 & Fire & 29 & 12.45 \\
\hline 4 & Sprain & 17 & 7.30 \\
\hline 5 & Skin allergy & 14 & 6.01 \\
\hline 6 & Toothache & 12 & 5.15 \\
\hline 7 & Conjunctivitis & 10 & 4.29 \\
\hline 8 & Gastroenteritis & 9 & 3.86 \\
\hline 9 & Stomachache & 8 & 3.43 \\
\hline 10 & Eye fatigue & 8 & 3.43 \\
\hline 11 & Dysmenorrhea & 7 & 3.01 \\
\hline 12 & Bronchitis & 5 & 2.15 \\
\hline 13 & Otitis media & 3 & 1.29 \\
\hline Total & & 233 & 100 \\
\hline
\end{tabular}

The 10 common diseases with a high proportion of distribution in autumn are cold, upper respiratory tract infections (pharyngitis, tonsils, etc.), fire, sprain, skin allergy, toothache, conjunctivitis, gastroenteritis, stomachache and eye fatigue, and their distribution proportions are shown in Fig 1.

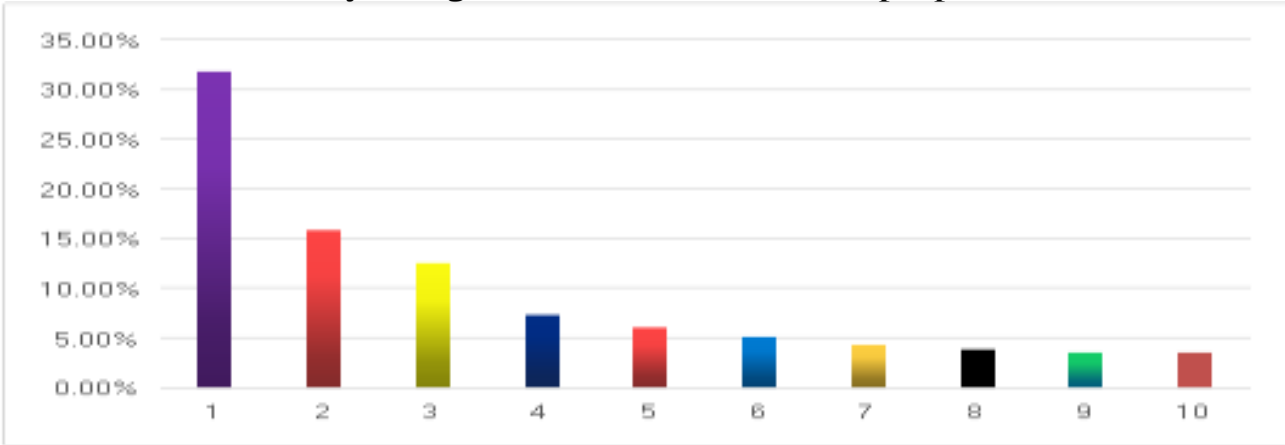

1.Cold 2.Upper respiratory tract infections (pharyngitis, tonsils, etc.) 3.Fire 4.Sprain 5.Skin allergy 6.Toothache 7.Conjunctivitis 8.Gastroenteritis 9.Stomachache 10.Eye fatigue

Figure 1. The distribution and proportion of college students' common diseases in autumn in Tianjin Agricultural University

\section{Statistical Analysis of College Students' Common Diseases in Winter in Tianjin Agricultural University}

From 16 November 2016 to 5 March 2017 (Winter), statistics were conducted on college students' common diseases treated by out-patients at Tianjin Agricultural University Hospital. The statistical results are shown in Table 2. In the winter, there are 218 common disease patients, accounting for more than $5 \%$ of the common diseases are cold, upper respiratory infections (pharyngitis, tonsils, etc.), fire, sprains, gastroenteritis, bronchitis and toothache. 
Table 2 The distribution and proportion of college students' common diseases in winter in Tianjin Agricultural University

\begin{tabular}{|c|c|c|c|}
\hline No. & Common diseases & Cases & Proportion[\%] \\
\hline 1 & Cold & 64 & 29.36 \\
\hline 2 & Upper respiratory tract infections (pharyngitis, tonsils, etc.) & 31 & 14.22 \\
\hline 3 & Fire & 23 & 10.55 \\
\hline 4 & Sprain & 21 & 9.63 \\
\hline 5 & Gastroenteritis & 17 & 7.80 \\
\hline 6 & Bronchitis & 15 & 6.88 \\
\hline 7 & Toothache & 14 & 6.42 \\
\hline 8 & Skin allergy & 11 & 4.59 \\
\hline 9 & Eye fatigue & 8 & 4.13 \\
\hline 10 & Stomachache & 6 & 2.75 \\
\hline 11 & Conjunctivitis & 5 & 2.29 \\
\hline 12 & Otitis media & 3 & 1.38 \\
\hline Total & & 218 & 100 \\
\hline
\end{tabular}

The 10 common diseases with a high proportion of distribution in winter are cold, upper respiratory tract infections (pharyngitis, tonsils, etc.), fire, sprain, gastroenteritis, bronchitis, toothache, skin allergy, eye fatigue and stomachache, and their distribution proportions are shown in Fig 2.

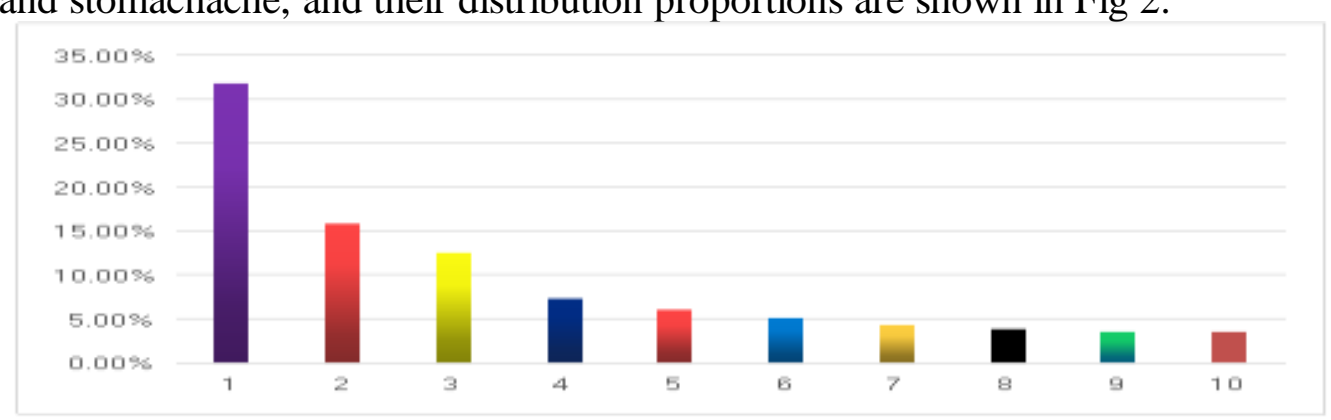

1.Cold 2.Upper respiratory tract infections (pharyngitis, tonsils, etc.) 3.Fire 4.Sprain 5.Gastroenteritis 6.Bronchitis 7.Toothache 8.Skin allergy 9.Eye fatigue 10.Stomachache

Figure 2. Distribution bar chart of 10 college students' common diseases in winter in Tianjin Agricultural University

\section{Statistical Analysis of College Students' Common Diseases in Spring in Tianjin Agricultural University}

From 6 March 2017 to 5 May 2017 (Spring), statistics were conducted on college students' common diseases treated by out-patients at Tianjin Agricultural University Hospital. The statistical results are shown in Table 3. In the spring, there are 364 common disease patients, accounting for more than $5 \%$ of the common diseases are cold, upper respiratory infections (pharyngitis, tonsils, etc.), sprains, fire, skin allergy and bronchitis. 
Table 3 The distribution and proportion of college students' common diseases in spring in Tianjin Agricultural University

\begin{tabular}{|c|c|c|c|}
\hline No. & Common diseases & Cases & Proportion[\%] \\
\hline 1 & Cold & 104 & 28.57 \\
\hline 2 & Upper respiratory tract infections (pharyngitis, tonsils, etc.) & 81 & 22.25 \\
\hline 3 & Sprain & 57 & 15.66 \\
\hline 4 & Fire & 29 & 7.97 \\
\hline 5 & Skin allergy & 20 & 5.49 \\
\hline 6 & Bronchitis & 19 & 5.22 \\
\hline 7 & Toothache & 13 & 3.57 \\
\hline 8 & Gastroenteritis & 12 & 3.30 \\
\hline 9 & Conjunctivitis & 11 & 3.02 \\
\hline 10 & Stomachache & 8 & 2.19 \\
\hline 11 & Beriberi & 6 & 1.64 \\
\hline 12 & Otitis media & 4 & 1.09 \\
\hline Total & & 364 & 100 \\
\hline
\end{tabular}

The 10 common diseases with a high proportion of distribution in spring are cold, upper respiratory tract infections (pharyngitis, tonsils, etc.), sprains, fire, skin allergy, bronchitis, toothache, gastroenteritis, conjunctivitis and stomachache, and their distribution proportions are shown in Fig 3.

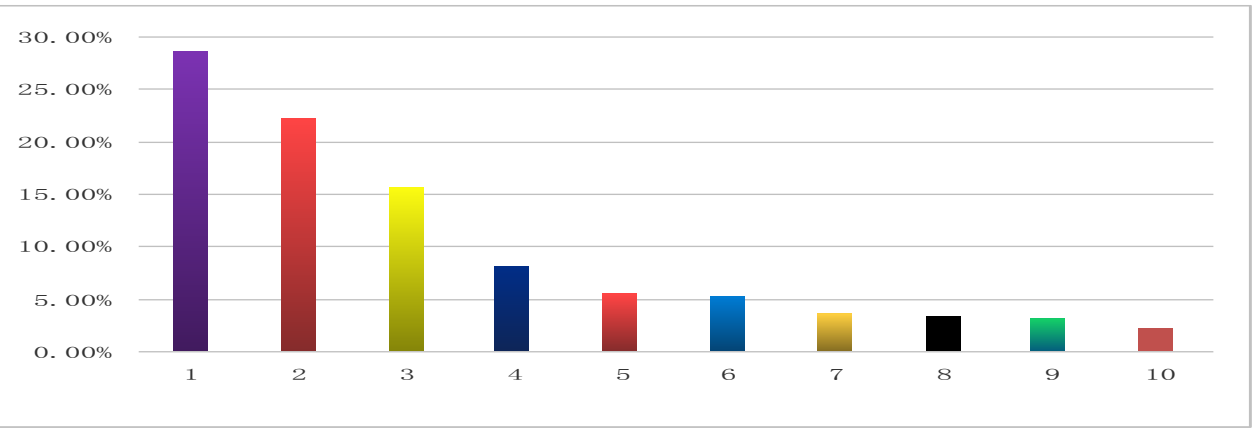

1.Cold 2.Upper respiratory tract infections (pharyngitis, tonsils, etc.) 3.Sprain 4.Fire 5.Skin allergy 6.Bronchitis 7.Toothache 8.Gastroenteritis 9.Conjunctivitis 10.Stomachache

Figure 3. Distribution bar chart of 10 college students' common diseases in spring in Tianjin Agricultural University

\section{Statistical Analysis of College Students' Common Diseases In Summer in Tianjin Agricultural University}

From 16 May 2017 to 15 September 2017 (Summer), statistics were conducted on college students' common diseases treated by out-patients at Tianjin Agricultural University Hospital. The statistical results are shown in Table 4 . In the summer, there are 260 common disease patients, accounting for more than $5 \%$ of the common diseases are cold, upper respiratory tract infections (pharyngitis, tonsils, etc.), sprain, skin allergy, gastroenteritis, fire and bronchitis. 
Table 4 The distribution and proportion of college students' common diseases in summer in Tianjin Agricultural University

\begin{tabular}{|c|c|c|c|}
\hline No. & Common diseases & Cases & Proportion[\%] \\
\hline 1 & Cold & 51 & 19.62 \\
\hline 2 & Upper respiratory tract infections (pharyngitis, tonsils, etc.) & 37 & 14.23 \\
\hline 3 & Sprain & 37 & 14.23 \\
\hline 4 & Skin allergy & 26 & 10.00 \\
\hline 5 & Gastroenteritis & 23 & 8.85 \\
\hline 6 & Fire & 22 & 8.46 \\
\hline 7 & Bronchitis & 14 & 5.38 \\
\hline 8 & Toothache & 12 & 4.62 \\
\hline 9 & Stomachache & 11 & 4.23 \\
\hline 10 & Conjunctivitis & 9 & 3.46 \\
\hline 11 & Headache,dizziness, hypertension and hypotension & 7 & 2.69 \\
\hline 12 & Dysmenorrhea & 6 & 2.40 \\
\hline 13 & Eye fatigue & 5 & 1.92 \\
\hline Total & & 260 & 100 \\
\hline
\end{tabular}

The 10 common diseases with a high proportion of distribution are cold, upper respiratory tract infections (pharyngitis, tonsils, etc.), sprain, skin allergy, gastroenteritis, fire, bronchitis, toothache, stomachache and conjunctivitis, and their distribution proportions are shown in Fig 4.

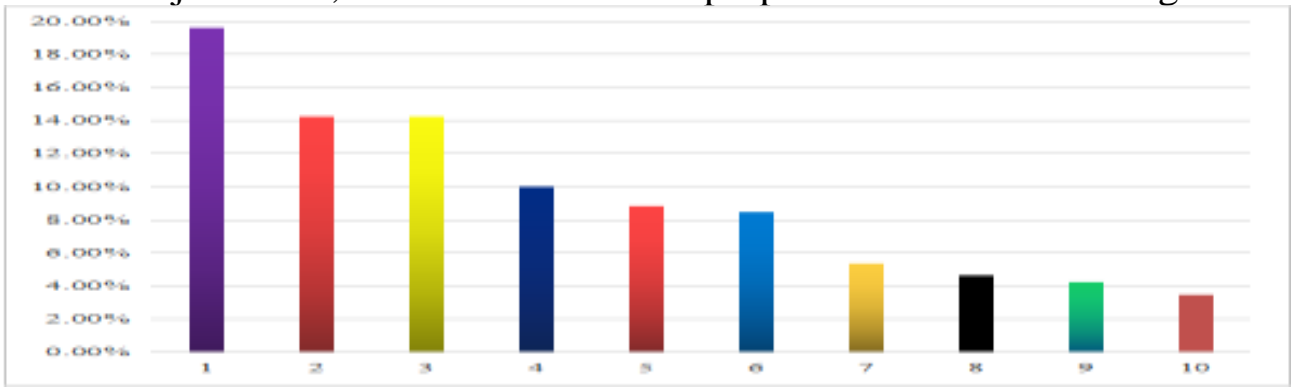

1.Cold 2.Upper respiratory tract infections (pharyngitis, tonsils, etc.) 3.Sprain 4.Skin allergy 5.Gastroenteritis 6.Fire 7.Bronchitis 8.Toothache 9.Stomachache 10.Conjunctivitis

Figure 4. Distribution bar chart of 10 college students' common diseases in summer in Tianjin Agricultural University

\section{Summary}

Through the statistical analysis of college students' common diseases in Tianjin Agricultural University by season, the number of common diseases in the spring (364 cases) is the highest and the incidence rate is the highest. Considering the time of winter and summer vacations, the incidence rate was higher in summer and winter, and the incidence was lowest in autumn (233 cases). The four most common diseases that have the highest incidence rate throughout the year are cold, upper respiratory tract infections (pharyngitis, tonsillitis, etc.), sprains and fire. The incidence of skin allergies was high in summer (10\%), autumn (6.01\%) and spring (5.49\%), and relatively low in winter (4.59\%). Gastroenteritis has a higher incidence in summer $(8.85 \%)$ and winter $(7.8 \%)$, and relatively low incidence in autumn (3.86\%) and spring (3.3\%). Tracheitis has a higher incidence in winter $(6.88 \%)$, summer $(5.38 \%)$, and spring $(5.22 \%)$, and a relatively low incidence in autumn $(2.15 \%)$. The incidence of toothache was high in winter $(6.42 \%)$ and autumn $(5.15 \%)$, and relatively low in summer $(4.62 \%)$ and spring (3.57\%). The incidence of conjunctivitis was high in autumn (4.29\%) and spring (3.02\%), and relatively low in summer $(2.69 \%)$ and winter $(2.29 \%)$. The incidence of stomachache was high in summer $(4.23 \%)$ and autumn (3.43\%), and relatively low in winter (2.75\%) and spring (2.19\%). Eye fatigue was higher in winter (4.13\%) and autumn (3.43\%), and lower in summer $(1.92 \%)$. 


\section{Acknowledgements}

This work was financially supported by the Project of Tianjin Agricultural University Education and Teaching Reform Research (2016-A-04).

\section{References}

[1] W.Y. Yan, X.M. Jia: For all Health, Vol. 10 (2016) No.15, p.8.

[2] Q.B. Wang, X. Shi, Y.Y. Wei: China Medical Herald, Vol. 6 (2009) No.26, p.120.

[3] X.C. Sun: China Medical Engineering, Vol. 19 (2011) No.12, p.149-150.

[4] W.J. Liu: Journal of Guangdong University of Petrochemical Technology, Vol. 23 (2013) No.5, p.35-37.

[5] Z,H, Lu: Journal of Qiqihar University of Medical, Vol. 36 (2015) No.11, p.1664-1665.

[6] Y.H. Feng, M.Y. Wu, H.L. Chen: Journal Traditional Medicine Management, Vol. 24 (2016) No.10, p.167-169.

[7] S.M. Sun: Chin J Sch Health, Vol. 35 (2014) No.11, p.1739-1741.

[8] Q.T. Wu, J.W. Gan: Health Medicine Research and Practice, Vol. 12 (2015) No.5, p.86-88. 